\title{
PROCESOS DE FORMACIÓN NATURALES ACTUANTES EN EL CASO DEL PECIO DE ZENCITY
}

\author{
María Teresita de Haro*
}

\begin{abstract}
Resumen
Durante los movimientos de suelo realizados en Puerto Madero para la construcción del complejo ZenCity ${ }^{1}$, en diciembre de 2008, quedaron al descubierto los restos de la estructura de lo que fuera un barco. Tras el aviso a las autoridades, el pecio ${ }^{2}$ fue rescatado por un equipo interdisciplinario al tiempo que se continuaba con la construcción. El registro hallado estaba conformado por la estructura del barco y una variedad de objetos pertenecientes al cargamento de la embarcación junto con otro grupo de materiales que no pertenecían al pecio pero sí conformaban el registro arqueológico. Presentaremos aquí el análisis de los posibles agentes naturales que habrían actuado en la formación del sitio.
\end{abstract}

Palabras claves: Pecio, contexto arqueológico, procesos de formación.

\begin{abstract}
During ground movements made in Puerto Madero to build, the ZenCity complex1 in December 2008, there were uncovered the remains of the structure of what once was a ship. After noticing the authorities, an interdisciplinary team rescued the shipwreck, meanwhile the construction continued. The register founded was conformed of the ship's structure and a variety of objects belonging to the cargo of the ship along with another group of materials, which did not belong to the shipwreck, but formed the final archaeological context. We present an analysis of the possible natural agents who have acted in the formation of the site here.
\end{abstract}

Key words: shipwreck, archaeological context, formation processes.

\section{Introducción}

En diciembre de 2008, durante la construcción del complejo ZenCity, en la zona de Puerto Madero, Ciudad Autónoma de Buenos Aires, Argentina, se desenterraron los restos de un barco o pecio (Figura 1). La empresa contratista dio aviso a las autoridades y la Dirección de Patrimonio e Instituto Histórico de la Ciudad Autónoma de Buenos Aires (D.G.P. e I.H. C.A.B.A.), institución a la que le correspondía hacerse cargo de la situación. Rápidamente la dirección convocó a un equipo interdisciplinario que, en convivencia con la obra de construcción, llevó a cabo el rescate del pecio. Adyacente a la estructura del barco se descubrieron una variedad de objetos pertenecientes al cargamento de la embarcación y otro conjunto de objetos

\footnotetext{
* Centro de Investigaciones Precolombinas. marite_dh@hotmail.com
} 
que no pertenecían al él, pero sí conformaban el contexto arqueológico (Valentini 2012).

Los restos descubiertos pertenecen a un barco de aproximadamente 30 metros de eslora $(28 \mathrm{~m})^{3}$, con una bodega que ocupa toda la extensión bajo cubierta, para un óptimo aprovechamiento de su capacidad de carga. La madera de roble y las características constructivas nos hablan de un mercante español del S XVIII, construido por un pequeño astillero de la ribera, en la zona del Cantábrico (García Cano 2012).
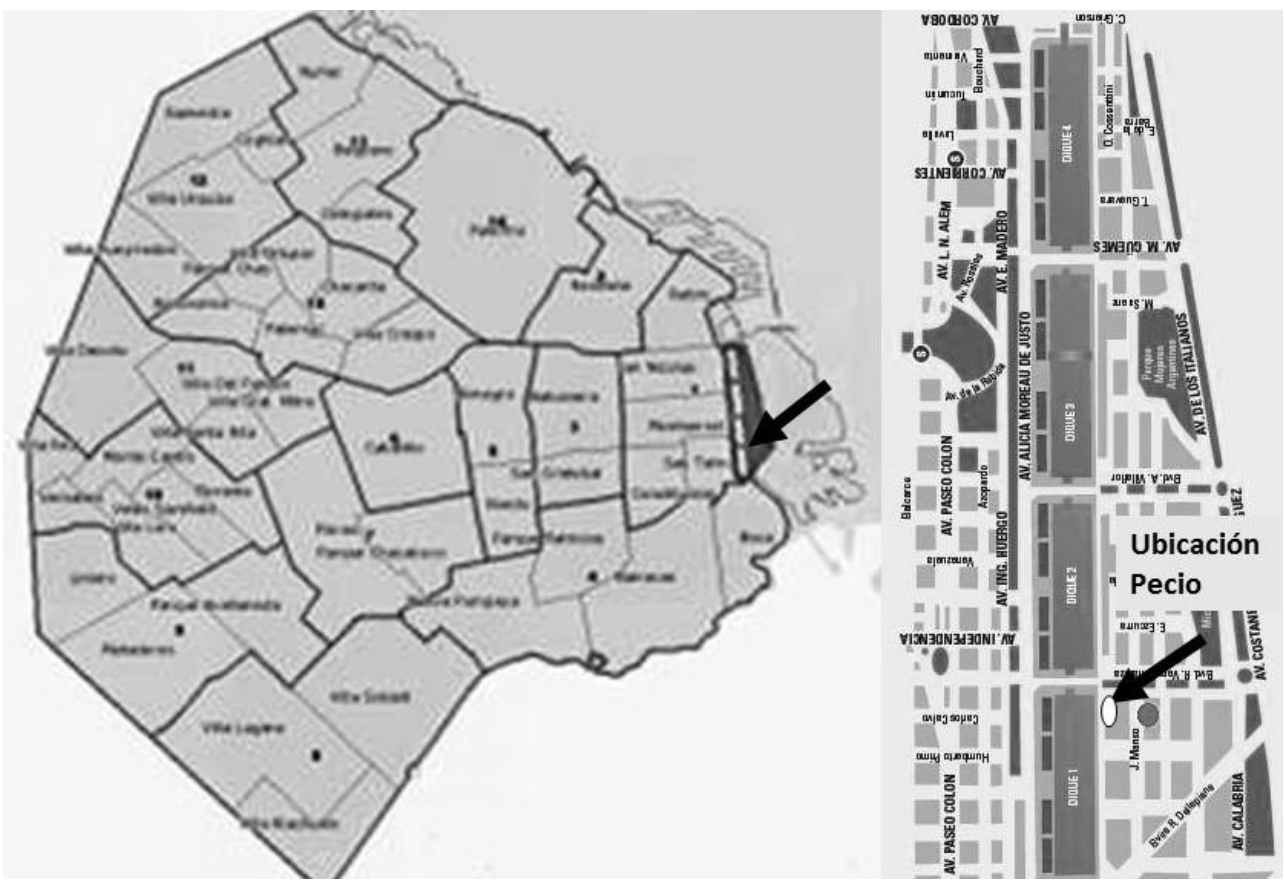

Figura 1. Ubicación del Pecio en el mapa de la Ciudad Autónoma de Buenos Aires. (www.buenosaires54.com/espanol/puertomadero.htm y www.buenosaireshabitat.com)

\section{¿De aquí o de allá?}

De acuerdo a las observaciones preliminares se pudo determinar que junto con la estructura del barco existían una variedad de objetos pertenecientes al cargamento de la embarcación (partes constitutivas de la estructura del barco, cañones, botijas y alcarrazas almacenadas bajo cubierta, zunchos y clavos pertenecientes a barriles, entre otros) un segundo grupo, cuya procedencia podía ser del barco mismo o no (fragmentos diversos de cerámicas vidriadas, mayólicas y lozas, materiales de construcción y fragmentos de cazoletas de pipas) y un tercer conjunto de objetos que claramente no pertenecían a la embarcación (material óseo y cerámica de adscripción hispano indígena o criolla, indígena y afroamericana), pero sí conformaban el registro arqueológico. 
Con respecto a los materiales cerámicos sí podemos asegurar, por ejemplo, que la gran colección de contenedores cerámicos o botijos que se hallaban en la bodega pertenecían a la carga del barco. En todo el mundo el comercio de ultramar utilizó estos contenedores cerámicos, en diferentes tamaños. Se utilizaron para el traslado de diversas mercancías, tales como aceite, vinagre, licores, granoso, olivas, etc..., por su eficacia para mantener los productos en buenas condiciones durante el traslado. Particularmente España hizo un uso intensivo de los mismos, se las suele encontrar citados en las listas de carga de tráfico marino entre la Casa de Contratación y las Colonias Americanas (López, 1980; Pasinsky y Fournier 2014).

Sin embargo hay otros objetos de uso común de cerámica vidriada, loza o mayólica o incluso cerámica rústica sin vidriado, cazoletas de pipas y materiales de construcción, de los cuales se encontraron fragmentos en menor cantidad que pueden tanto pertenecer al barco como provenir de fuera de él. Las lozas procedían generalmente de Europa y en particular, en mayor medida, de Inglaterra, sobre todo dentro del marco temporal del que estamos hablando. La mayólica también era corrientemente de proveniencia europea, mayoritariamente española, aunque en América se las fabricaba también en diversos sitios de México, Perú, Guatemala y Panamá desde el S XVI (Schávelzon, 2001).

En cuanto a los materiales de construcción, se fabricaban ladrillos en forma local desde aproximadamente el 1600 y tejas y baldosas un poco más tarde. De todas formas aunque se fabricaran en América también se continuó con la importación de tejas y baldosas, desde España y Portugal para la época estudiada y desde Francia hacia finales del S XIX.

En relación a la cerámica no vidriada, se trata de un conjunto heterogéneo, al cual se denominó cerámica local, debido a que se trata, la gran mayoría, de fragmentos identificables como cerámica de confección indígena, hispano-americana o criolla y afro-americana. Algunos pocos fragmentos, por su confección en torno, podrían ser importados. Dicho grupo se encuentra en este momento en etapa de estudio, sin embargo es detectable a simple vista que los fragmentos han tenido diferentes historias de vida con respecto a los procesos de formación que los afectaron.

Consideramos al Registro Arqueológico como la consecuencia residual de una enorme serie de interacciones de procesos naturales y culturales que ocurren en un amplio rango de escalas de tiempo espacial, temporal y conductual. Es creado por comportamientos antrópicos y faunisticos y disturbado por procesos postdeposicionales comprobados teórica y metodológicamente (Heilen et al. 2008). Según Binford (1988:23), el modo de encontrarle un sentido al Registro Arqueológico hallado es "averiguando cómo llegaron a existir esos materiales, cómo se han modificado y cómo adquirieron las características que vemos hoy". Se trata de analizar cuáles fueron las circunstancias y los agentes que participaron en el paso de los objetos desde su contexto sistémico al contexto de hallazgo (Schiffer, 1990). A su vez es a través de la observación de los procesos de formación y con una mirada que abarque no solamente al sitio en sí, sino a todo su entorno, que podremos analizar la aparición de estos otros componentes que se fueron sumando al contexto primigenio. 


\section{Procesos de Formación}

El estudio de los procesos de formación incluye la investigación tanto de los Procesos de formación medioambientales o naturales como deterioro, desgaste, procesos de perturbación medioambiental, y procesos de superficie de la tierra (pátinas, deterioro fúngico, crioturbación y vulcanismo); como de los Procesos de formación culturales que incluyen reutilización, descarte, reclamación y perturbación cultural (Heilen et al. 2008). Por otra parte, si consideramos que, los paisajes son constructos culturales, y son una interacción dinámica entre naturaleza y cultura a través del tiempo, un enfoque del paisaje complementa el análisis espacio-temporal tradicional de la arqueología, mediante sus medios de análisis científicos y procesuales, a la vez que, al mismo tiempo integra la historia y los actos humanos en sus construcciones (Anchuetz et al. 2001). Se presentarán aquí los Procesos de Formación Naturales.

\section{Procesos de Formación Medioambientales o Naturales}

En este caso se analizarán los procesos de formación naturales que actuaron. En la región Pampeana son identificables, a grandes rasgos, tres unidades geomórficas:

1- Planicie Pampeana, en este sector se perciben los Sedimentos Pampeanos que fueron depositados por la acción eólica, modelados por los agentes fluviales. Limitada al este y noreste por un Paleoacantilado.

2- Planicie Estuárica actual, ubicada hacia el este del delta del río Paraná, a lo largo de la zona costera, con ambientes de acumulación de litorales. En la zona de la Ciudad Autónoma de Buenos Aires se halla totalmente modificada.

3- Delta del río Paraná, conformado por acumulaciones fluviales.

Por otra parte, la fracción interior de la Plataforma Continental Bonaerense en la zona del Río de La Plata, denominada Terraza Rioplatense, presenta una superficie irregular debido a la existencia de cordones de playa, dunas y escalones erosivos y se encuentra atravesada por el paleocauce del Rio de la Plata (Canal Oriental). Es una franja sumergida desde el frente de costa hasta el pie de un escalón ubicado a 70 metros de profundidad, constituida por arenas de características variables. Además, se aprecian unidades geomorfológicas de menor orden, tales como el Alto marítimo, el Banco La Plata, la Restinga de los Pescadores, el Sistema de Bancos Alineados, el Canal Oriental y otras (Iriondo, 2010), todas estas formaciones han sido, a lo largo del tiempo, una "trampa" aún para los marineros más avezados. Frente al Río de La Plata se localiza una línea de costa de forma alargada del tipo "barrera", rica en arena biodetrítica y conchas enteras y fragmentadas (Parker y Violante, 1992).

El río de la Plata es un acentuado golfo de origen tectónico en el cual convergen las aguas dulces de los ríos Paraná y Uruguay (Viana Maturro, 2009). Su longitud es de alrededor de $300 \mathrm{~km}$ con un ancho mínimo de $40 \mathrm{~km}$ y un máximo de $200 \mathrm{~km}$ en su desembocadura, donde vierte sus aguas al océano sin barrera alguna (Boschi 1987). Con una geometría y batimetría complejas, en la zona interna es muy 
somero con profundidades que oscilan entre 1.0 y $3.0 \mathrm{~m}$, mientras que en el límite externo llega a $18 \mathrm{~m}$ de profundidad (FREPLATA 2014). Existen canales de navegación y bajos producidos por las mismas corrientes de los cuales, en la zona norte, el Canal Oriental el más profundo del sistema, con profundidades de hasta $25 \mathrm{~m}$, se extiende a lo largo de la costa uruguaya. Su reducida profundidad media no le permite generar sus propias mareas, por lo cual los cambios de nivel de sus aguas se deben a la influencia del océano y a las perturbaciones atmosféricas que pueden alterar marcadamente el desplazamiento de las aguas (Boschi 1987). Actualmente las características del Río de la Plata, simultáneamente fluviales y estuáricas, son consecuencia de la interacción de factores hídricos y climáticos con la dinámica de los sedimentos y su historia geológica. Posee un sistema estuarino donde se mezclan las aguas dulces de sus tributarios con las saladas provenientes del Océano Atlántico (Boschi, 1987; Cavallotto y Violante, 2011).

Los sedimentos que llegan al Río de la Plata provienen casi en su totalidad del río Paraná (principalmente Paraná de las Palmas y Paraná Guazú), que descarga sus aguas en forma de un gran delta altamente constructivo, conformando una costa altamente progradante (Iriondo y Scotta, 1978; Marcomini y López, 2004; FREPLATA 2014 y Figura 2). Estudios efectuados durante los últimos 100 años han demostrado que su régimen de flujo es de una gran variabilidad, con una componente cuasi-decádica y picos de variabilidad inter-anual en las escalas temporales asociadas al fenómeno de El Niño-Oscilación del Sur (ENOS), esto deriva en periodos de grandes bajas en su altitud intercalados con otros de crecidas de considerable magnitud (Camilloni, 2014; FREPLATA, 2014). La composición sedimentaria nos habla de una historia muy compleja de un sistema que posee, aún al día de hoy, una dinámica muy activa (Iriondo 1985).

Figura 2. Sentido en que el río Paraná descarga los sedimentos hacia el Estuario del Río de la Plata y vista más detallada del comportamiento del flujo del agua en el estuario (Marcomini y López 2004)

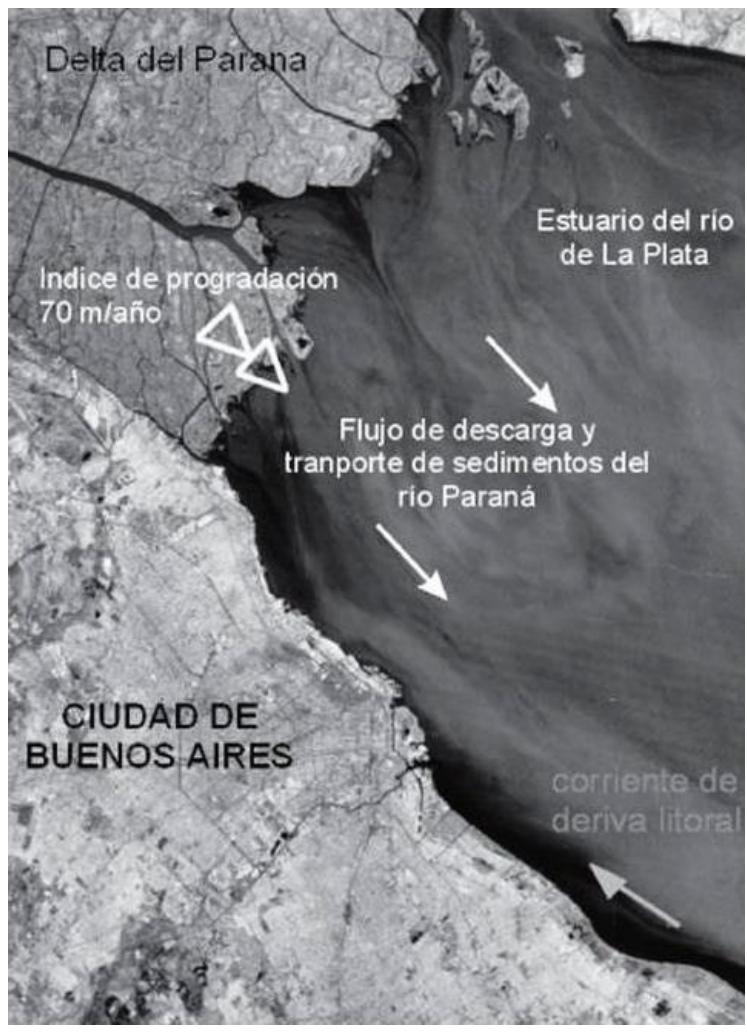


Es importante dentro de estos procesos tener conocimiento del comportamiento del agua y los sedimentos. Las corrientes mueven los sedimentos diferencialmente, siendo que los que se hallan más cerca de la superficie se mueven más que los que están situados en el fondo Tarbuck y Lutgens 1999). El transporte de sedimentos, por la acción del flujo, puede desarrollarse en forma de arrastre de fondo, en suspensión y como carga de lavado (Brea y Spalletti, 2010).

Los sedimentos son depositados cuando la velocidad de la corriente se ralentiza, denominándose estos depósitos fluviales como aluviones, pudiendo ser éstos depósitos de canal o barras, o de llanura de inundación tales como diques naturales, deltas o abanicos aluviales en las desembocaduras (Tarbuck y Lutgens 1999). Por otra parte, la velocidad del agua y por consiguiente su tensión de arrastre es máxima hacia el centro de la sección del cauce y mínima en las proximidades del perímetro del cauce (Acuapedia 2011). En el caso de los ríos de valle y los que desembocan en el mar, la poca pendiente ocasiona la reducción de la velocidad del caudal generando que los componentes de los sedimentos sean de grano fino, también debido a una movilidad diferencial que relaciona el tamaño de las partículas en movimiento con la velocidad de la corriente, ya que la velocidad mínima para desprender y transportar una partícula de suelo está en función del diámetro de ésta (García-Chevesich). En el Río de La Plata se evidencia una disminución de energía de la corriente a medida que el agua avanza, demostrada por la calidad de los sedimentos (limos y arcillas) (Senciales González, 1998).

Otra cuestión a tener en cuenta es la turbulencia generada en el flujo del agua cuando un objeto se interpones en ella y se produce un efecto de separación del fluido. Esta separación ocasiona, por un lado, que las partículas de agua se "aplasten" contra la superficie que les presenta resistencia y circulen a mayor velocidad en los perfiles del objeto y, por otro, se genera un área de turbulencia, denominada estela, en la porción distal del objeto, donde las partículas vuelven a unirse. En sistemas de baja velocidad, puede hasta producirse un retroceso de los sedimentos en dicho sector. Cabe decir que la presión ejercida por la corriente puede generar deformaciones en el cuerpo y al igual que el área de turbulencia depende de la forma de éste (Dalmati 1962 y Figura 3).

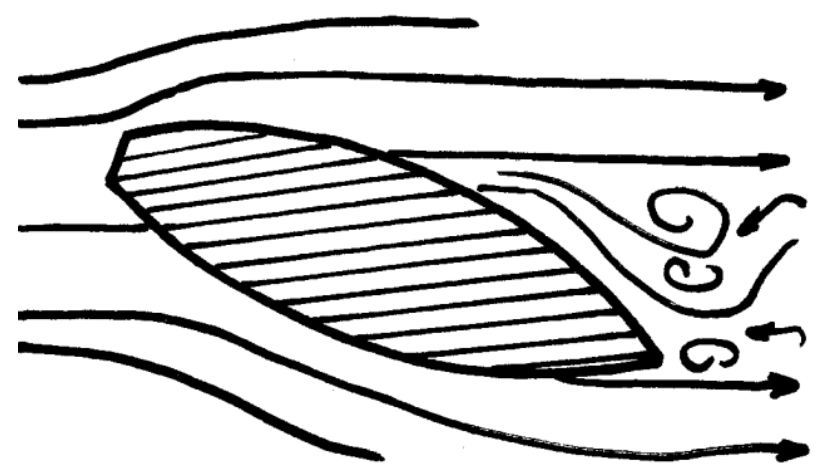

Figura 3. Estela formada tras un objeto fluidodinámico modificado de Dalmati 1962 
Esta misma dinámica es la que convierte a cualquier cuerpo, situado estáticamente en el camino de la corriente de un río, en un elemento de atracción de todo objeto que esté siendo arrastrado por las aguas en las cercanías. Cuando dicho cuerpo se encuentra en los márgenes del curso de agua, donde menor es su fuerza de arrastre, mayor es la capacidad de "captación" que posee.

$\mathrm{Si}$ bien las acciones de los animales (peces, aves, microorganismos, roedores) y de la vegetación (algas, juncos, etc.), pueden haber actuado como modificadores bióticos del sitio, no se ha incluido en este trabajo su análisis.

\section{Conclusión}

Por ser el Río de La Plata un río con mucho arrastre de sedimentos que forman múltiples bancos de arena, es probable que el barco quedara varado en las proximidades de la orilla debido a dichos bancos y/o barras, luego de haber tenido una avería que lo imposibilitó para seguir navegando (Valentini et al. 2012). Al momento del rescate se hallaron, dentro de él y/o en sus proximidades elementos que no eran parte de lo que denominamos el contexto primario del evento, sino que configuraban un contexto secundario, fruto de la acumulación de objetos a causa de diversos factores, parte de ellos fueron los Procesos de Formación Naturales.

Debido al efecto causado por la turbulencia del agua, los restos del Pecio se comportaron a lo largo del tiempo como un "imán", atrayendo aquellos objetos que eran transportados junto con los sedimentos, en suspensión o por el fondo. Cabe mencionar que parte de los sedimentos que arrastraba el río pudieron ser los desprendidos de la propia barranca de la ciudad de Buenos Aires. También influyeron en este proceso las contracorrientes generadas por las sudestadas y la influencia de las mareas en el estuario. Completando los procesos de formación naturales de sitio podemos citar las diferencias de cota del río debido a diversos periodos climáticos generados por el Fenómeno del Niño, que influyeron en el nivel de las aguas del estuario pudiendo dejar por momentos al descubierto los restos, mientras que en otras temporadas los tapaban por completo (Deschamps y Tonni, 2007 y 2009).

\section{Notas}

1. Complejo de edificios, oficinas, viviendas, centro comercial, hotel y cocheras subterráneas.

2. Lámase pecio a los restos materiales producto del naufragio de un barco.

3. No se posee la medida exacta, ya que la estructura no está completa debido a las obras de excavación que, por un lado, la sacaron a la luz y, por otro, destruyeron parte de la misma. (Valentini, comunicación personal).

\section{Bibliografía}

AGUAPEDIA, 2011. Morfología y Dinámica fluvial. http://www.prueba2.aguapedia.org/master/ponencias/modulo6/morfología (acceso 5 marzo 2015). 
ANCHUETZ, K.F., R.H. WILSHUSEN Y C.L. SCHEICK, 2001. An Archaeology of Landscapes: perpectives andirections. Journal of Archaeological Research, 9 (2): 157-211.

BINFORD, L. 1998 (1988). En busca del pasado. Crítica. Barcelona.

BORRAZO, K. 2006. Tafonomía Litica en las dunas: una propuesta para el análisis de los artefactos líticos. Intersecciones en Antropología. 7:247-261.

BOSCHI E. 1987. El ecosistema estuarial del Río de la Plata (Argentina y Uruguay). Anales del Instituto de Ciencias del mar y Limnología. http://biblioweb.tic.unam.mx/cienciasdelmar/instituto/1988-2/articulos313.html (acceso 15 de marzo de 2015).

BREA, J.D. Y P.D. SPALLETTI, 2010. Generación y transporte de sedimentos en cuencas de ríos de montaña. Procesos de erosión-sedimentación cauces y cuencas. Documeneto Técnico. Programa Hidrológico Internacional UNESCO pp. 52-74. Barlocchi y Brea eds. Montevideo.

CAMILLIONI, I. 2014. Variabilidad y tendencias hidrológicas en la Cuenca del Plata. El cambio climático en el Río de la Plata pp.21-32. CONICET-UBA. Vicente Barros Ed. Buenos Aires.

DALMATI, D. 1962. Manual de Hidrología. Cap. IV. Vol 1. Centro de Estudiantes de Ingeniería de La Plata. La Plata.

DESCHAMPS, J. Y TONNI, E. 2007. Aspectos ambientales en torno al primer fuerte sur de Buenos Aires: "El Zanjón 1745-1779. Universidad de Belgrano. Buenos Aires. http://www.ub.edu...ar/invetigaciones/dt_nuevos/175_deschamps.pdf (acceso 21 de diciembre de 2014).

FREPLATA 2014 Manual-PHC-FFEM. Manual freplata: Estudio de la Dinámica Hidro-sedimentológica del Río de la Plata: observación y modelación numérica de los sedimentos finos. Buenos Aires. http://www.ina.gov.ar PROYECTO FREPLATA RLA 99/G31. CONVENIO DE COOPERACIÓN N CZZ1268.01 (acceso 20 de noviembre de 2014).

GARCÍA CANO, J. 2012. ¿Por qué un mercante español? Un mercante español en el puerto de Buenos Aires. Historias y hallazgos en Puerto Madero pp. 24-31. CDROM. M. Valentini, J. García Cano, \& Compiladores. Dirección General de Patrimonio e Instituto Histórico. Buenos Aires.

GARCÍA-CHEVESICH, P. 2010. Factores que afectan la erosión y la sedimentación. Procesos de erosión - sedimentaciónen cauces y cuencas. Documento Técnico 
No22. Vol. 1: 9-18. UNESCO. Programa Hidrológico Internacional. Barlocchi-Brea Ed. Montevideo. http://eias.utalca.cl/isi/publicaciones/erosion_y_sedimentacion_vol1.pdf (acceso 15 de mayo de 2015).

HEILEN, M.P., M.B. SCHIFFER Y J. JEFFERSON REID. 2008. Landscape Formation Processes. Handbook of landscape archaeology pp. 601-608. D. Bruno, \& J. Thomas Editores. Left Coast Press. Walnut Creek.

HISCOCK, P. 1985. The need for taphonomic perpective in stone artefact analysis. Queensland Archaeological Research 2: 82-95.

IRIONDO, M. 1985. Los sedimentos del subsuelo del delta del río Paraná. Editorial Universidad Nacional del Litoral. Santa Fe.

2010. Geología del cuaternario en Argentina Vol. 3. Sudamérica, Grupo de Estudio del Cuaternario (GEC). Paraná.

IRIONDO, M. Y SCOTTA, E. 1978. The evolution of the Paraná River delta. International Symposium on Coastal Evolution in the Quaternary - Proceedings pp 405418. Y. Suguio, Ed.

LÓPEZ, S. 1980. Arqueoloxia sobmariña: os materais procedentes da badia coruñesa. Brigatium I: 139-165.

MARCOMINI SILVIA Y RUBÉN LÓPEZ. 2004. Revista de la Asociación Geológica Argentina, 59(2): 261- 272.

OLIVER, J. 2005. Comentarios en torno a la temprana introducción y uso de algunas plantas domésticas, silvestres y cultivos en Las Antillas precolombinas de Jaime Pagán Jiménez et al. Diálogo Antropológico 3(10): 35-41.

OZÁN, I. 2010. Procesos de formación en cerámica de cazadores recolectores de ls provincia de La Pampa: alcances y limitaciones de una experimentación. Boletín del Laboratorio de Petrología y Conservación Cerámica 2(2): 9-17. http://arqueologia.unca.edu.ar/assets/boletin-lpcc-vol2-n2-a (acceso 2 de julio de 2010).

PASINSKY, T y P. FOURNIER. S/f Ceramics: The Ibero-American Shipping Container. http//link.springer.com.ezproxy2.library.arizona.edu/referenceworkentry/10.1007/978-1 (acceso 07 de febrero de 2014).

REID, K. 1984. Fire and ice: new evidence for the production and preservation of late archaic fiber-tempered pottery in the midle-latitude lowlands. American Antiquity 49(1): 72-76. 
SCHÁVELZON, D. 2001. Catálogo de cerámicas históricas de Buenos Aires (Siglos XVI-XX). Con notas sobre la región del Río de La Plata CD-ROM. Telefónica-Centro de Arqueología Urbana-Fundación para la investigación del Arte Argentino-Facultad de Arquitectura, Diseño y Urbanismo, Universidad de Buenos Aires. Buenos Aires.

SCHIFFER, M. 1990. Contexto arqueológico y contexto sistémico. Boletín de Antropología Americana 22: 81- 93.

SENCIALES GONZÁLEZ, J. M. 1998. El analisis morfologico de las Cuencas Fluviales aplicado al Estudio Hidrográfico. Norba. Revista de Geografía 10: 155-183. http://dialnet.unirioja.es/servlet/articulo?codigo=109746 (accedido 11 de mayo de 2015).

TARBUCK, E. Y F. LUTGENS. 1999. Ciencias de la tierra. Prentice Hall. Madrid.

VALENTINI, M. 2012. El registro arqueológico. Arqueología de un barco. Un mercante español en el puerto de Buenos Aires. Historias y hallazgos en Puerto Madero pp. 33-37. CD-ROM. M. Valentini, J. García Cano, \& Compiladores. Dirección General de Patrimonio e Instituto Histórico. Buenos Aires.

VALENTINI, M., J. GARCÍA CANO Y M. FERNÁNDEZ. 2012. Aproximaciones al conocimiento del Pecio de ZenCity. Anti 11: 57- 62.

VIANA MATURRO, F. 2009. www.ecoplata.org. http://www.ecoplata.org/wp-content/files_mf/2009 zonacosteradeluruguaybiodiversidadygestion.pdf (acceso $28 \mathrm{de}$ marzo de 2015).

Sitios web:

www.buenosaires54.com/espanol/puertomadero.htm

www.buenosaireshabitat.com

Recibido: 14 de abril 2016.

Aceptado: 22 de septiembre 2016. 